\title{
Ratchet Effect and Nonlinear Transport for Particles on Random Substrates with Crossed ac Drives
}

\author{
C. Reichhardt and C.J. Olson Reichhardt \\ Center for Nonlinear Studies and Theoretical Division, Los Alamos National Laboratory, Los Alamos, New Mexico 87545
}

(November 19, 2018)

\begin{abstract}
We show in simulations that overdamped interacting particles in two dimensions with a randomly disordered substrate can exhibit novel nonequilibrium transport phenomena including a transverse ratchet effect, where a combined dc drive and circular ac drive produce a drift velocity in the direction transverse to the applied dc drive. The random disorder does not break any global symmetry; however, in two dimensions, symmetry breaking occurs due to the chirality of the circular drive. In addition to inducing the transverse ratchet effect, increasing the ac amplitude also strongly affects the longitudinal velocity response and can produce what we term an overshoot effect where the longitudinal dc velocity is higher in the presence of the ac drive than it would be for a dc drive alone. We also find a dynamical reordering transition upon increasing the ac amplitude. In the absence of a dc drive, it is possible to obtain a ratchet effect when the combined ac drives produce particle orbits that break a reflection symmetry. In this case, as the ac amplitude increases, current reversals can occur. These effects may be observable for vortices in type II superconductors as well as for colloids interacting with random substrates.
\end{abstract}

PACS numbers: 05.60.-k, 05.45.-a, 74.25.Qt

\section{INTRODUCTION}

There has been growing interest in studying nonequilibrium transport of particles on asymmetric substrates in which a dc flow of particles can arise from the application of an ac drive or by flashing the substrate on and off periodically [1]. This rectification phenomena is often called a ratchet effect and has been studied in the context of molecular motors [2], colloidal matter [3], transport of atoms in optical traps [4], granular matter [5], electron transport in asymmetric geometries [6], vortex transport and manipulation in type-II superconductors [7-12], and transport in Josephson junctions $[13,14]$. In most of these systems the dc transport arises due to the symmetry breaking caused by an imposed one-dimensional asymmetric substrate, such as an asymmetric sawtooth potential. In higher dimensions, there are a larger number of possible ways in which symmetry can be broken, so it is possible to induce ratchet effects even when the substrate is symmetric.

Recently it was shown that a driven overdamped particle moving in a two-dimensional periodic substrate can exhibit a transverse ratchet effect when driven with both a dc and a circular ac drive [15]. In this case, the chirality of the ac drive breaks a symmetry and the dc drive breaks a reflection symmetry, so that the combined effect is to induce an additional dc particle current in the direction perpendicular to the applied external dc drive. Due to the periodicity of the substrate and the periodicity of the ac driving, a series of phase locking steps appear in both the longitudinal velocity and the transverse velocity. Even more complicated transport phenomena occur when the ac drive is not circular but elliptical [16]. In this case it is possible to observe a phenomenon called absolute transverse mobility, where the particle moves only perpendicular to an applied dc drive.

Recently, a rich variety of ratchet dynamics were shown to occur for atoms driven over a two-dimensional substrate with crossed or biharmonic ac drives [17]. It has also been shown that a ratchet effect can be produced even in the absence of a dc drive for an overdamped particle in a two-dimensional periodic potential when the crossed ac drives produce a particle orbit that breaks a spatial reflection symmetry [16]. In this case a series of phase locked regions appear as a function of ac amplitude. In all these cases the substrate is periodic and the symmetry breaking required to produce the ratchet effect arises from the ac drives. An open question is whether a net dc current or ratchet effect can occur for driven particles on random substrates when subjected to a circular ac drive. Large scale vorticity patterns were observed in simulations of particles interacting with random substrates when an ac drive was applied in only one direction [18]. The large scale flow arises since random substrates can cause a local symmetry breaking, giving rise to a local ratchet effect. There is no net global ratchet effect since the symmetry is restored on average at large length scales. These results suggest that ratchet effects should be possible in dimensions higher than one even when the substrate is random.

A ratchet effect that occurs on random substrates could be of great practical importance since many systems in which applications for a ratchet effect have been proposed contain intrinsic random disorder. If a ratchet effect could be produced directly from the random disorder, additional fabrication of structured substrates would 
not be needed. One example of such a system is vortices in type II superconductors, where random defects act as pinning sites. Ratchets previously proposed for this system have all included periodic substrates of some form [7-12]; however, the random substrate ratchet effect we study here could be achievable experimentally without nanostructuring the superconducting surface.

In this work we show that several types of ratchet and nonequilibrium transport phenomena can occur for particles interacting with a two-dimensional random substrates with crossed ac driving forces. In the absence of the disordered substrate there is no ratchet effect. For the case where there is a circular ac drive and an applied dc drive, a transverse ratchet effect can occur in which a net dc drift arises in the direction perpendicular to the dc drive. If the ac drives are chosen such that the particles undergo spatially asymmetric orbits, directed transport can occur even the absence of the applied dc drive. It is also possible to have flux reversals as a function of ac amplitude. In the absence of disorder, the multiple ac drives do not produce a ratchet effect. We specifically demonstrate these ratchet effects for vortices in type-II superconductors and colloidal particles confined to two dimensions.

\section{SIMULATION}

We consider an assembly of $N_{v}$ overdamped interacting particles in two dimensions, and impose periodic boundary conditions in the $x$ and $y$ directions. The equation of motion for an individual particle $i$ is

$$
\eta \mathbf{v}_{i}=\mathbf{f}_{i}=\mathbf{f}_{i n t}^{i}+\mathbf{f}_{p}^{i}+\mathbf{f}_{d c}+\mathbf{f}_{a c}+\mathbf{f}_{i}^{T}
$$

where the damping constant $\eta$ is set to unity. The equation of motion is integrated according to the leapfrog method with a normalized time step $d t=0.001$. The interaction force from the other particles is $\mathbf{f}_{i n t}^{i}=$ $-\sum_{j \neq i}^{N_{v}} \nabla_{i} U\left(r_{i j}\right)$, where $r_{i j}$ is the distance between particles $i$ and $j$. We consider two forms of particle-particle interactions. Vortices in a thin film type II superconductor have a repulsive interaction with $U\left(r_{i j}\right)=-\ln \left(r_{i j}\right)$ which gives a long range repulsive force $1 / r_{i j}$. We have previously simulated vortices in periodic arrays of pinning sites using this interaction and a similar equation of motion. In order to treat the long-range interactions efficiently, we employ a fast summation technique [19]. Additionally we have considered a short range repulsive Yukawa interaction with inverse screening length $\kappa$, $U\left(r_{i j}\right)=\exp \left(-\kappa r_{i j}\right) / r_{i j}$, which is appropriate for colloidal particles. In this case, we assume that we are working in the strongly charged, low volume fraction limit, so that hydrodynamic interactions can be neglected.

The force from the random substrate is modeled as $N_{p}$ randomly placed attractive parabolic wells with
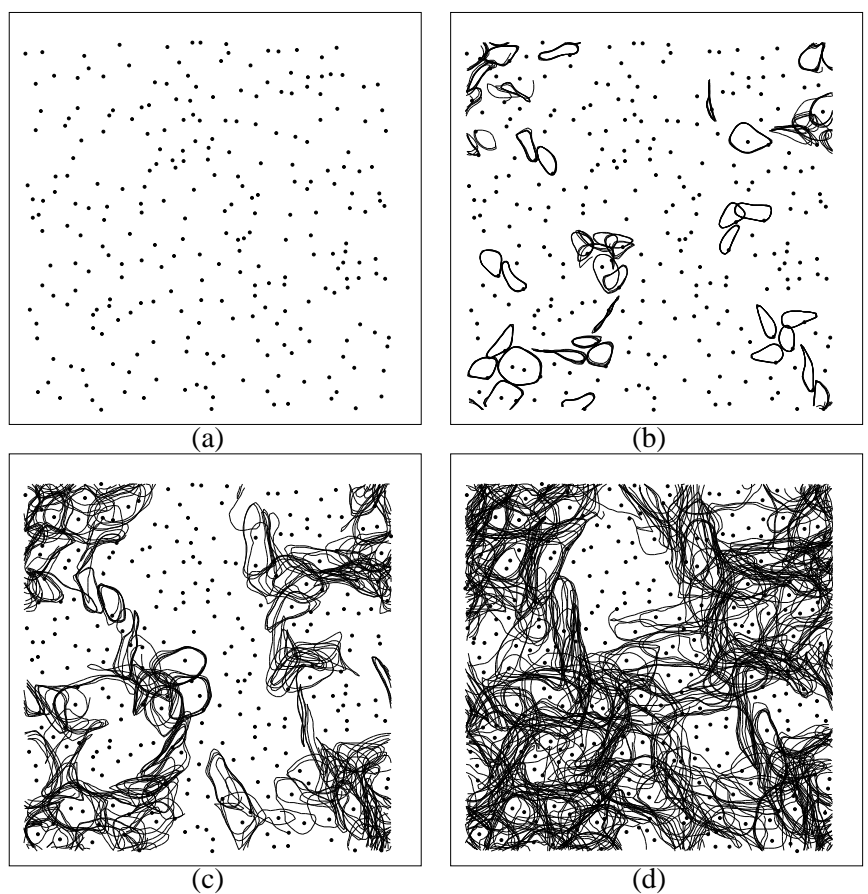

(d)

FIG. 1. Particles (black dots) and trajectories (black lines) for a fixed period of time for a system with random pinning and a circular ac drive with fixed frequency $\omega=0.001$ and increasing amplitude $A$. (a) $A=0.0$, (b) $A=0.5$, (c) $A=0.8$, and (d) $A=1.2$.

$$
\mathbf{f}_{p}^{i}=-\sum_{k=1}^{N_{p}}\left(f_{p} / r_{p}\right)\left|\mathbf{r}_{i}-\mathbf{r}_{k}^{(p)}\right| \Theta\left(r_{p}-\left|\mathbf{r}_{i}-\mathbf{r}_{k}^{(p)}\right|\right) \hat{\mathbf{r}}_{i k}^{(p)}
$$

where $\Theta$ is the Heaviside step function, $\mathbf{r}_{k}^{(p)}$ is the location of pinning site $k, f_{p}$ is the maximum pinning force, $r_{p}$ is the pinning site radius and $\hat{\mathbf{r}}_{i k}^{(p)}=\left(\mathbf{r}_{i}-\mathbf{r}_{k}^{(p)}\right) /\left|\mathbf{r}_{i}-\mathbf{r}_{k}^{(p)}\right|$. The force from the temperature $\mathbf{f}_{i}^{T}$ is modeled as random Langevin kicks with the properties $\left\langle f_{i}^{T}(t)\right\rangle=0$ and $\left\langle f_{i}^{T}(t) f_{j}^{T}\left(t^{\prime}\right)\right\rangle=2 \eta k_{B} T \delta_{i j} \delta\left(t-t^{\prime}\right)$. Except where noted, the results are obtained at $T=0$. In some cases we apply a dc force, $\mathbf{f}_{d c}$, which is modeled as a uniform force on all the particles in the $x$ direction. In this work we increase the dc force in small increments, and we have checked that the force increase is small enough that transient effects are negligible. The ac drive force is

$$
\mathbf{f}_{a c}=f(t) \hat{\mathbf{x}}+g(t) \hat{\mathbf{y}} .
$$

Here $f(t)$ and $g(t)$ are oscillating functions with $\langle f(t)\rangle_{\tau}=$ 0 and $\langle g(t)\rangle_{\tau}=0.0$ over a period $\tau$. In the first part of this work we will consider the circular drive case of $f(t)=A \sin (\omega t)$ and $g(t)=A \cos (\omega t)$. We measure the particle trajectories and velocities in the longitudinal $\left\langle V_{x}\right\rangle=\left(1 / N_{v}\right)\left\langle\sum_{i}^{N_{v}} \hat{\mathbf{x}} \cdot \mathbf{v}_{i}\right\rangle$ and transverse direction $\left\langle V_{y}\right\rangle=\left(1 / N_{v}\right)\left\langle\sum_{i}^{N_{v}} \hat{\mathbf{y}} \cdot \mathbf{v}_{i}\right\rangle$. We average over several hundred periods of the ac drive in order to obtain a steady state average. 


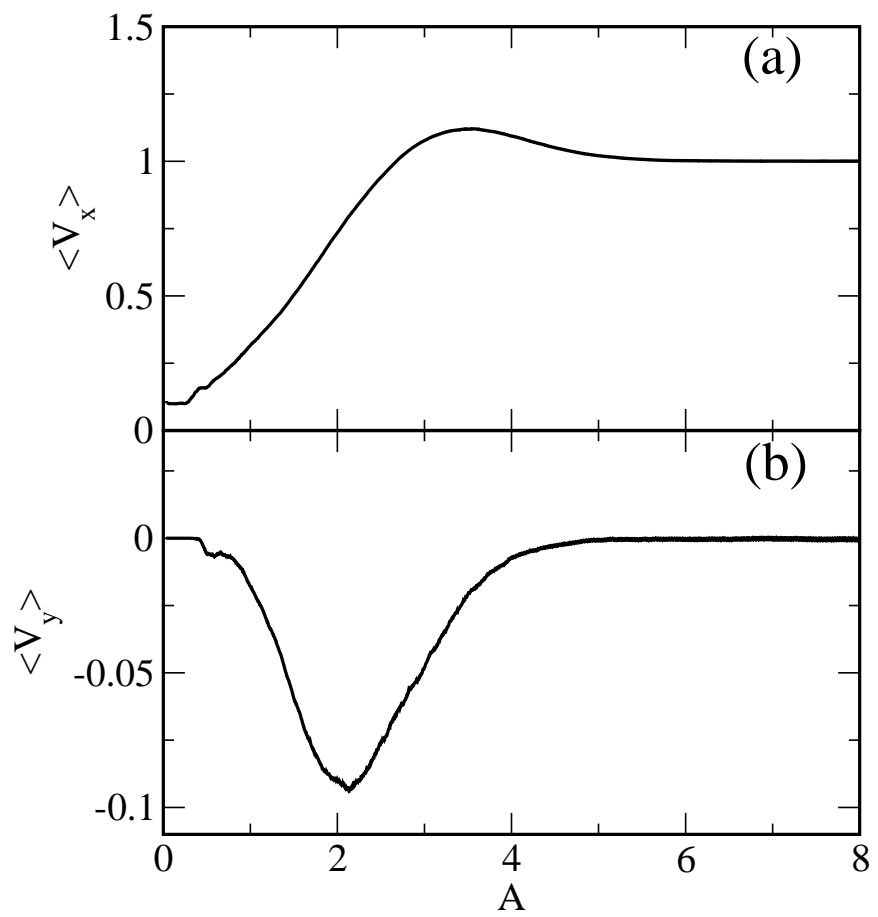

FIG. 2. The average velocity response in the (a) $x$ and (b) $y$ directions for a system with $f_{p}=3.5$, a fixed dc drive of $f_{d c}=1.0$, and increasing circular ac drive amplitude $A$.

\section{CIRCULAR AC DRIVES AND TRANSVERSE RECTIFICATION}

\section{A. Transverse and Longitudinal Velocity-Force Characteristics}

We first consider the case of a circular ac drive and no dc drive. In Fig. 1 we illustrate the particle motion over a fixed period of time for a system with $N_{p}=250, N_{v}=$ $280, f_{p}=3.5, T=0.0, f_{d c}=0$, fixed frequency $\omega=$ 0.001 , and an increasing ac amplitude $A$. In Fig. 1(a), $A=0.0$ and all the particles are stationary. In Fig. 1(b), at $A=0.5$, a portion of the particles are pinned and do not move with the ac drive; however, several particles are now mobile and follow closed paths. In the absence of other particles the paths would be circular, but due to the repulsion of the neighboring trapped particles, the paths are strongly distorted from circular shapes. In general, at low ac amplitude some of the particles move in closed paths but there is no long time particle diffusion. If there are many more particles than pinning sites, $N_{v} \gg N_{p}$, it is possible for meandering paths that change with time to form, permitting some particles to diffuse throughout the entire sample over long time scales. When the number of particles is close to or less than the number of pinning sites, $N_{v} \lesssim N_{p}$, similar meandering paths can only form at

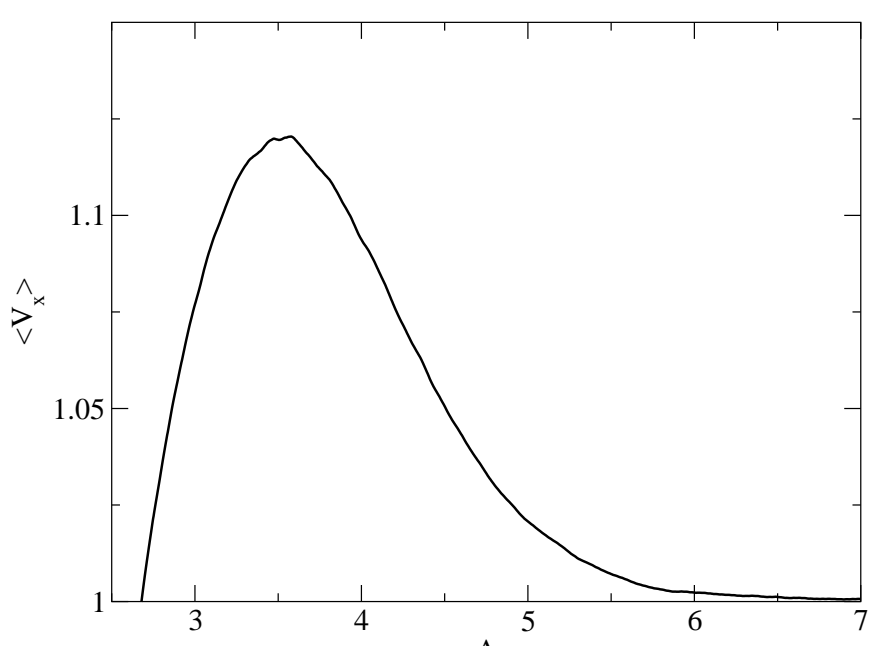

FIG. 3. A closeup of $\left\langle V_{x}\right\rangle$ vs $A$ A for the system in Fig. 2(a) showing the overshoot region where $\left\langle V_{x}\right\rangle>1.0$.

higher ac amplitudes when pinning and repinning of particles at the pinning sites becomes possible, as shown in Fig. 1(c) for $A=0.8$. As the ac amplitude is further increased, more of the particles become mobile and eventually all the particles depin. In Fig. 1(d) we show the case for $A=1.2$ where large portions of the particles are depinned and the channel structures in which motion is occurring change rapidly with time.

In the absence of a dc drive, a purely circular ac drive does not produce a net drift velocity, and $\left\langle V_{x}\right\rangle=\left\langle V_{y}\right\rangle=$ 0 . If the system size is very small and finite size effects become relevant, it is possible to observe a small dc drift. For the system sizes we consider here, these finite size effects are eliminated. In Fig. 2 we plot $\left\langle V_{x}\right\rangle$ and $\left\langle V_{y}\right\rangle$ vs $A$ for the same system in Fig. 1 with a fixed dc drive $f_{d c}=1.0$ applied in the $x$ direction. In the absence of pinning, a single particle subjected to this combination of drives would move in the $x$-direction with $\left\langle V_{x}\right\rangle=1.0$. For $A=0$, most of the particles are pinned, $\left\langle V_{x}\right\rangle=0.1$, and $\left\langle V_{y}\right\rangle=0$. For $A<0.58$, there is little change in $\left\langle V_{x}\right\rangle$ and $\left\langle V_{y}\right\rangle$, while for $0.58<A<2.0$ the velocity in the $x$-direction increases, indicating that more particles are depinning and moving in the direction of the $\mathrm{dc}$ drive. Over this same range of $A,\left\langle V_{y}\right\rangle$ drops below zero and develops an increasingly negative value, indicating that particles are drifting in the negative $y$-direction in spite of the fact that there is no applied dc drive in the $y$ direction. The transverse velocity reaches a maximum magnitude $\left|\left\langle V_{y}\right\rangle\right|=0.09$ near $A=2.1$ and then gradually returns to zero at $A \sim 5$. For $A>6$ the longitudinal velocity saturates at $\left\langle V_{x}\right\rangle=1.0$ indicating that the pinning has been washed out and the particles are moving at the same velocity as they would in the absence of pinning. For $2.6<A<6$, in a range falling above the maximum of $\left|\left\langle V_{y}\right\rangle\right|$ but below the saturation of $\left\langle V_{x}\right\rangle$, there is what we term an overshoot effect where the longitudinal velocity 


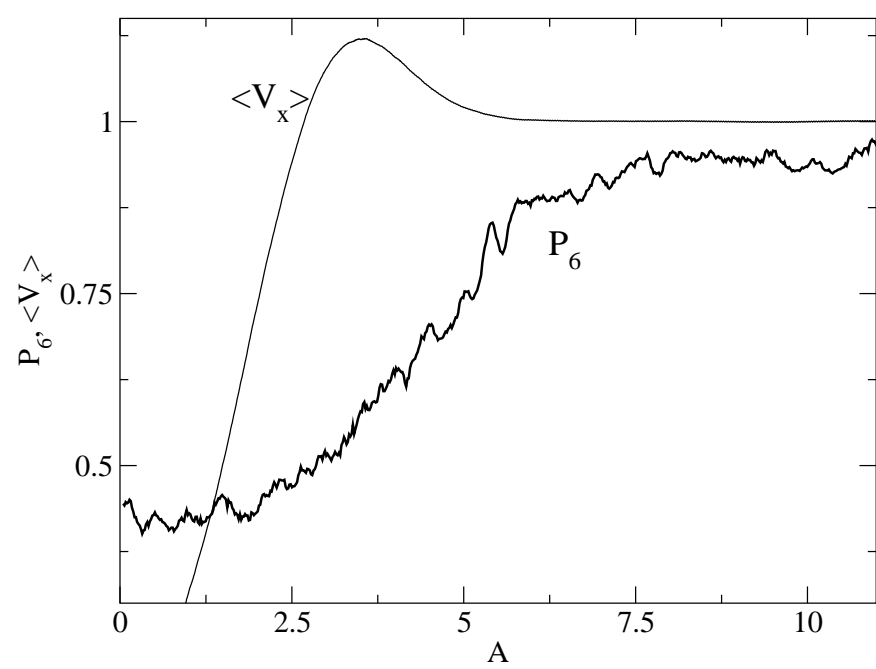

FIG. 4. The fraction of six-fold coordinated particles $P_{6}$ vs $A$ for the same system in Fig. 2. $\left\langle V_{x}\right\rangle$ vs $A$ is also plotted for direct comparison to show that the overshoot effect is lost when the lattice reorders.

$\left\langle V_{x}\right\rangle$ is greater than the maximum possible velocity expected for the applied dc drive, $\left\langle V_{x}\right\rangle>1.0$. In Fig. 3(a) we show a blowup of this region where there is a peak value of $\left\langle V_{x}\right\rangle=1.12$ at $A=3.5$, giving an excess velocity of 0.12 . This implies that some of the energy from the $x$ component of the ac drive is being coupled into the particle motion during the positive $x$ direction half of the ac cycle, but an equivalent amount of energy is not being removed during the negative $x$ direction half of the ac cycle, producing a net imbalance. The random substrate plays an important role in this effect, as indicated by the fact that $\left\langle V_{x}\right\rangle$ gradually drops back to a saturation value of 1.0 as the ac drive amplitude is further increased and washes out the effect of the pinning.

The transverse ratchet and the overshoot effect can both be understood as arising from the combination of the symmetry breaking from the circular drive and the nonlinear features of the velocity-force curves that appear when plasticity is induced as particles are driven over a random substrate, as we will demonstrate in the following sections. In general, for particles such as vortices or colloids moving over strong random disorder under the influence of a dc drive $f$, there are three distinct dynamic regimes [20-23]. For low drives $f$, all the particles are pinned, and there is a threshold depinning force $f_{c}$ that must be applied before motion begins. As the driving is increased further above depinning, $f \gtrsim f_{c}$, there is a plastic flow regime where pinned and flowing particles coexist. In this regime the velocity vs force curves are nonlinear and can be fit to a power law form $V=\left(f-f_{c}\right)^{\beta}$. For elastic depinning, $\beta=2 / 3$, but for the plastic depinning that occurs for strongly disordered substrates, $\beta>1.0[9,25,26]$. At even higher drives, a dynamical reordering effect occurs in which the particles

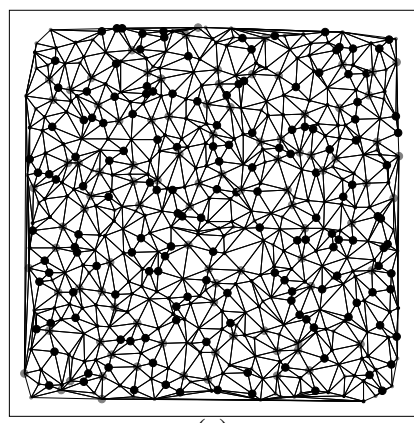

(a)

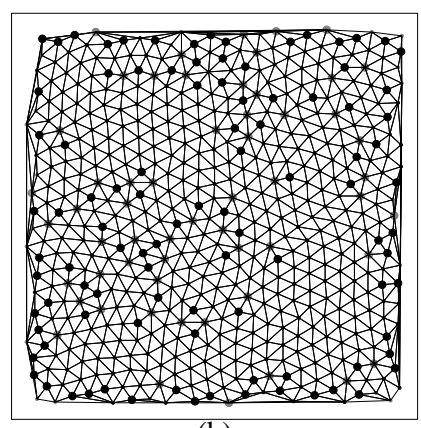

(b)

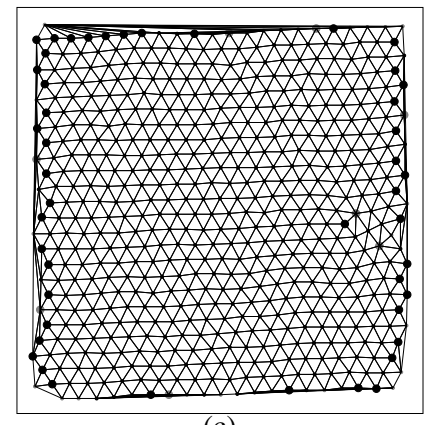

(c)

FIG. 5. The Delaunay triangulation of the particle positions at one instant for different values of $A$ for the system in Fig. 4. The large black dots indicate fivefold coordinated particles and the lighter large dots indicate sevenfold coordinated particles. (a) $A=0.5$, (b) $A=2.5$, and (c) $A=10$.

crystallize or partially crystallize when the longitudinal component of the pinning is effectively washed out. Above this transition, the velocity force curves are linear. The reordered state can be a moving crystal or moving smectic state [23]. When these three phases occur, the velocity force curves have a distinct S-shape, and the concavity of the velocity force curve changes from positive to negative somewhere in the plastic flow regime as the plastic to moving crystal crossover is approached. In the crossover regime that occurs above the change in concavity, $\beta$ drops from $\beta>1$ to $\beta=1.0$.

\section{B. Dynamic Reordering with Circular ac Drives}

If a single ac drive is applied in the $x$ direction instead of a dc drive, it is still possible to produce the three dynamic regimes described above as a function of ac amplitude and frequency since the transitions between the regimes are not hysteretic, and the system will reorder into a moving smectic state [27]. In our system we apply a pair of ac drives to produce a circular ac drive, rather than a single unidirectional ac drive, and we also add a dc drive. We find that a dynamical reordering transition can still occur in this system, but that the final reordered state is not a moving smectic for small $f_{d c}$ since the ac drive does not select a particular direction. In Fig. 4 we plot the fraction of six-fold coordinated particles $P_{6}$ vs $A$ 
for the same system shown in Figs. 2 and 3, and we also superimpose $\left\langle V_{x}\right\rangle$ from Fig. 2(a) for a direct comparison. The quantity $P_{6}$ is obtained from a Delaunay triangulation of the system. If the entire lattice is triangular, then $P_{6}=1.0$. For low $A$ the system is strongly disordered, as indicated by the fact that $P_{6} \sim 0.4$. As $A$ is increased, $P_{6}$ increases rapidly toward one for $A>2.5$, which also corresponds to the region in which the overshoot effect of $\left\langle V_{x}\right\rangle$ occurs. For high values of $A>6$, the system is reordered and $P_{6}$ is close to one as the system enters a moving crystal phase. We note that $P_{6}$ never reaches one due to the boundary effects on the algorithm we used to obtain the coordination number. When $P_{6}$ saturates, the overshoot effect and the transverse ratchet effect are both lost.

In Fig. 5 we illustrate the Delaunay triangulations of the particles at increasing values of $A$ for the system in Fig. 4. In Fig. 5(a) for $A=0.5$, the system is strongly disordered. In Fig. 5(b) for $A=2.5$, the system is more ordered and contains regions of sixfold coordinated particles. For $A=10$, shown in Fig. 5(c), the system is almost completely reordered into a triangular lattice and only a small number of dislocations are present. We note that the lattice is not well aligned with the direction of the dc drive, but has its close-packed direction at an angle to the $x$ axis. The near alignment occurs due to the fact that a small dc drive is being applied. When we perform the simulation with zero dc drive, the reordering still occurs but the direction of the lattice alignment is random in any given run. This is in contrast with previous simulations performed with dc drives [22] where the reordered lattices formed moving channels that were strongly aligned with the dc drive. In the case of reordering induced by a purely dc drive, the dislocations present at higher drives all have aligned Burgers vectors and the system forms a smectic state since the driving force has reduced the pinning only in the direction of drive, but the pinning transverse to the driving direction still remains effective [23]. In our ac driven system, the one-dimensional channel structures of the smectic state cannot form since the circular ac motion destroys the effectiveness of the transverse pinning as well as of the longitudinal pinning. Thus we find no moving smectic state.

\section{Transverse Ratchet and Overshoot Effect}

Under the circular ac drive, each particle has a nonzero velocity component in the positive $y$ direction for half a period, and in the negative $y$ direction for the other half period. There is no dc force applied in the $y$ direction so these components are equal in magnitude. For the velocity component in the $x$ direction, during half the period $0<t^{*}<\tau / 2$, where $t^{*}=t \bmod \tau$, the ac and $\mathrm{dc}$ drives are in the same direction and the particles move faster under the maximum force $f_{\max }=f_{d c}+A$, while in the other half of the period $\tau / 2<t^{*}<\tau$, the two drives counteract each other and the particle moves more slowly under the minimum force $f_{\min }=f_{d c}-A$. If we consider the limit of small ac driving amplitude, where the system is still within the nonlinear portion of the velocity-force response curve, a portion of the particles have depinned but a portion remain pinned. Under the drive we apply, the moving particles follow clockwise circles. During an orbit in which a moving particle encircles a pinned particle, the pinned particle exerts a repulsive force on the encircling particle. For $0<t^{*}<\tau / 2$, the moving particle passes the pinned particle in a short period of time so the repulsive force has less time to deflect the particle in the $+y$ direction. On the other hand, for $\tau / 2<t^{*}<\tau$, the moving particle takes longer to pass the pinned particle, so the average deflection in the $-y$ direction is larger. As $A$ increases from a small value, more particles become unpinned and the average velocity in the $-y$ direction increases. When $A$ is large enough that more than half the particles are depinned, the $-y$ ratcheting effect starts to decrease since there are fewer pinned particles available to deflect the paths of the moving particles. The velocity should go as the product of the fraction of moving particles $n_{m}$ and pinned particles $n_{p},\left\langle V_{y}\right\rangle=n_{m} n_{p}=n_{m}\left(1-n_{m}\right)$. This gives a parabolic shape for $\left\langle V_{y}\right\rangle$, which is similar to what is observed in Fig. 2(b), and the peak should occur when $n_{m}=1 / 2$. At the larger drives when all the particles are moving and the velocity-force curve is no longer nonlinear, the transverse ratcheting effect is lost. The maximum for $\left|\left\langle V_{y}\right\rangle\right|$ in Fig. 2 occurs at $A=2.0$. If we take the approximation that the unpinned particles move at the velocity of the applied dc drive, then $n_{m}=0.5$ would correspond to $\left\langle V_{x}\right\rangle=0.5$, which occurs at $A=1.5$, not far from the maximum in $\left|\left\langle V_{y}\right\rangle\right|$. At high $A$ when all the particles are depinned, the transverse drift disappears.

The overshoot effect can be understood as arising from changes in the nonlinear velocity force curve relations near the reordering transition. Well below the reordering transition, the velocity scales with the driving force as a power law $\left\langle V_{x}\right\rangle \propto A^{\beta_{1}}$, where $\beta_{1}>1$, but above the reordering transition $\left\langle V_{x}\right\rangle$ scales linearly with the velocity. As a result, near but below the reordering transition, the scaling relation changes form and the scaling exponent may be slightly different, giving $\left\langle V_{x}\right\rangle \propto A^{\beta_{2}}$, with $\beta_{2} \rightarrow 1$ as the reordering transition is approached. For $A$ near but below the reordering transition, when the ac and dc forces sum to $f_{\max }$ in the positive $x$ direction during the interval $0<t^{*}<\tau / 2$, the velocity follows $A^{\beta_{2}}$. During the other half of the cycle $\tau / 2<t^{*}<\tau$, the particle motion slows under $f_{\min }$ and the velocity follows $A^{\beta_{1}}$. The difference during a full period is $\Delta V_{x}(A) \approx A^{\beta_{1}}-A^{\beta_{2}}$. In the high driving regime where the velocity response is linear, $\beta_{1}=\beta_{2}=1.0$, so $\Delta V_{x}=0$. Near the ordering transition, $\beta_{2}$ is very close to 1 since it is very 


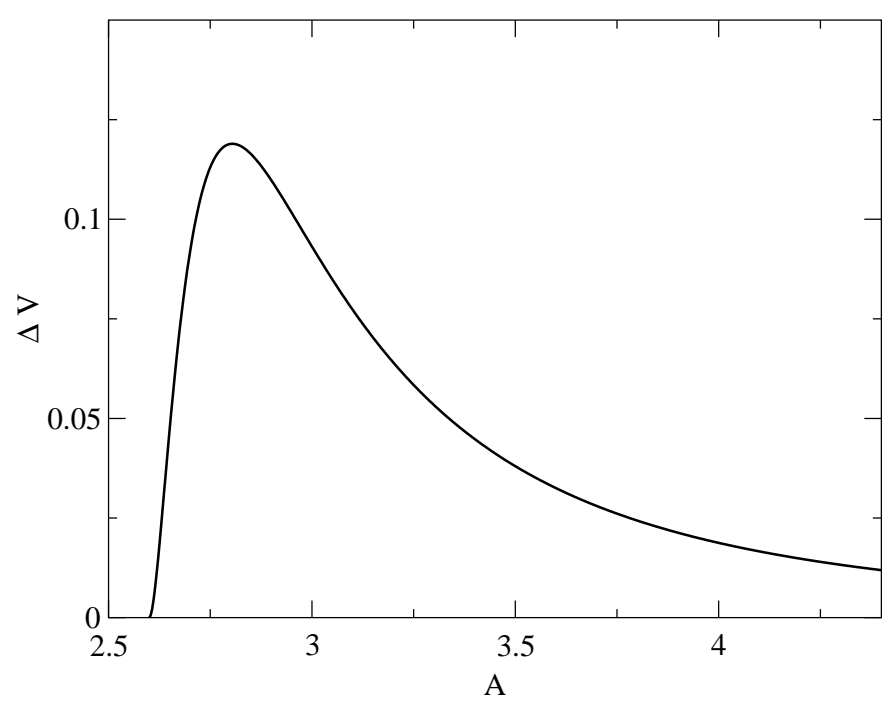

FIG. 6. Plot of the behavior of $\Delta V(A)$ vs $A$ from the phenomenological form $\Delta V(A)=B\left[A^{\left(1-(1 / A)^{\delta_{1}}\right)}-A^{\left(1-(1 / A)^{\delta_{2}}\right)}\right]+C$ for $\delta_{1}=4.0$, $\delta_{2}=3.9, B=19.8$, and $C=1.6$.

close to the region where the velocity force curve is linear. In contrast, $\beta_{1}>1.0$, so that $A^{\beta_{2}}<A^{\beta_{1}}$. As a result, there is a net positive contribution from the ac drive to the dc force over each cycle, giving rise to an average velocity $\left\langle V_{x}\right\rangle>1$, higher than the velocity produced by a strictly dc drive. We now make a very simple phenomenological assumption for the form of the excess velocity: $\Delta V(A)=A^{\left(1-(1 / A)^{\delta_{1}}\right)}-A^{\left(1-(1 / A)^{\delta_{2}}\right)}$. With this form, $\Delta V(A)=0$ for large $A$ when both exponents go to one. We also assume that $\delta_{1}$ is larger than $\delta_{2}$ by a small amount, so that the exponent on the $\tau / 2<t^{*}<\tau$ half of the cycle is always smaller than that on the $0<t^{*}<\tau / 2$ half of the cycle. For all $\delta_{1,2}>1$ and $A>1, \Delta V(A)$ shows a peak and a slow roll off to zero, illustrated in Fig. 6, which is very similar to the behavior of the excess velocity seen in Fig. 3. This suggests that the overshoot velocity arises due to the nonlinear form of the velocity force curves near the dynamic reordering transition.

\section{Effects of Disorder Strength, Particle Density, and Temperature}

We next examine how the transverse ratchet effect evolves as a function of disorder strength. In Fig. 7 we plot $\left\langle V_{y}\right\rangle$ vs $A$ for $f_{p}=0.5,2.5,3.5,4.5$, and 5.5. For the weak disorder $f_{p}=0.5$, we do not observe any transverse ratcheting, as indicated by the flat line in Fig. 7. As the disorder strength increases, the maximum value of $\left|\left\langle V_{y}\right\rangle\right|$ increases and the peak value shifts to higher $A$. The reordering transition at high $A$ also moves out to larger A. A similar trend appears in the longitudinal velocities $\left\langle V_{x}\right\rangle$ with the overshoot phenomenon showing

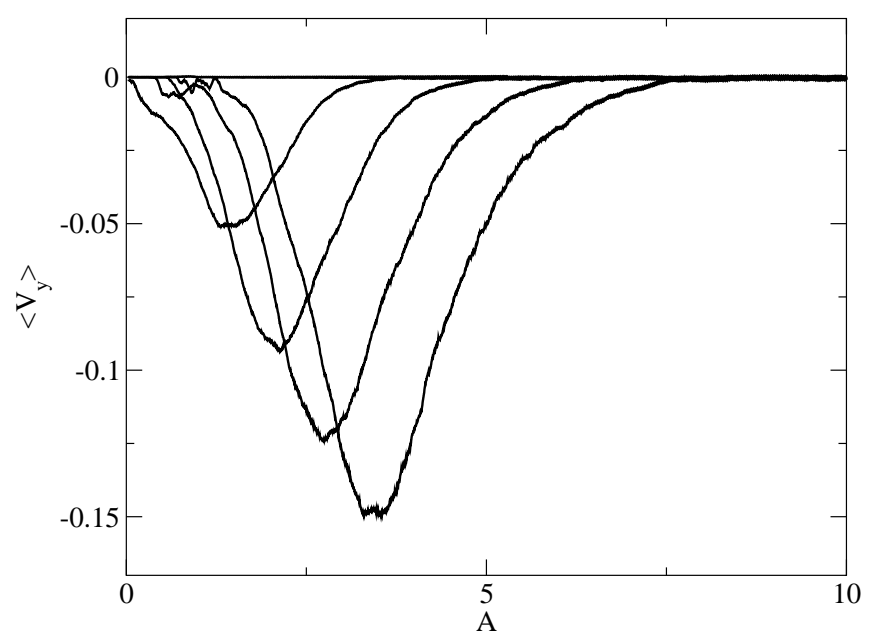

FIG. 7. $V_{y}$ vs $A$ for varied $f_{p}$ for the same system parameters as in Fig. 2. From top minimum to bottom minimum, $f_{p}=0.5$ (flat line), 2.5, 3.5, 4.5, and 5.5.

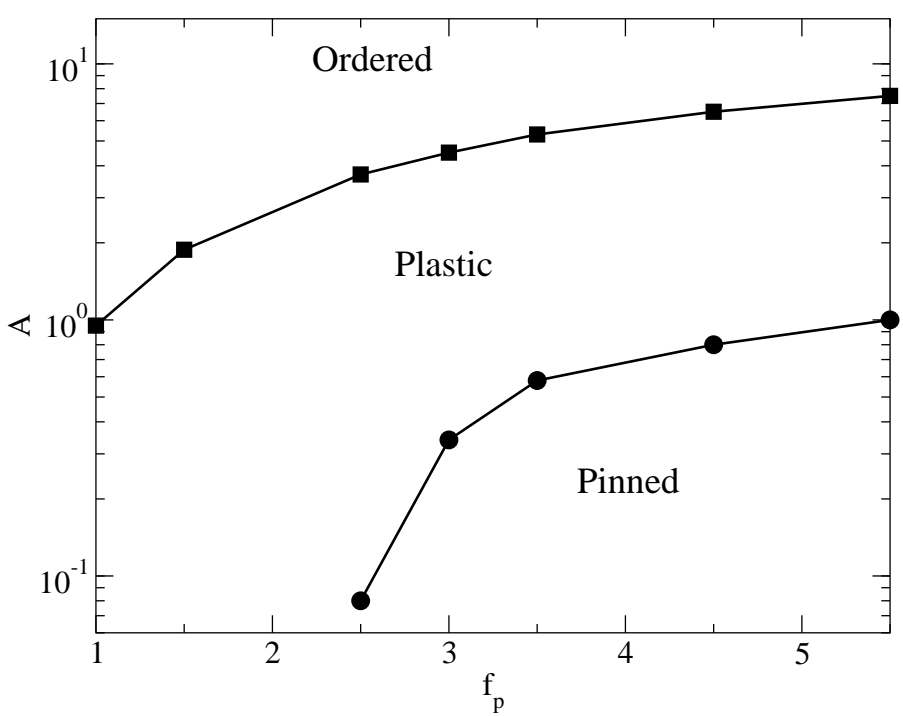

FIG. 8. Dynamic phase diagram as a function of $A$ and $f_{p}$ showing the pinned regime, plastic flow regime, and reordered regime. The reordered regime is similar to that illustrated in Fig. 5(c).

the same behavior as in Fig. 2(a). In Fig. 8 we map out the dynamic phase diagram for $A$ vs $f_{p}$. For high $A$ the system forms an ordered phase similar to that illustrated in Fig. 5(c). For low $A$ and high $f_{p}$ the system is in the pinned phase. We note that, due to the constant applied $f_{d}=1$, if $f_{p}<f_{d}$, the system passes directly into a moving phase and is never pinned. The intermediate regime is the plastic flow phase where there is a finite transverse velocity. As $f_{p}$ is lowered, the onset of the ordered phase drops to lower values of $A$. For $f_{p}<1.0$ the pinning is weak enough that large portions of the 

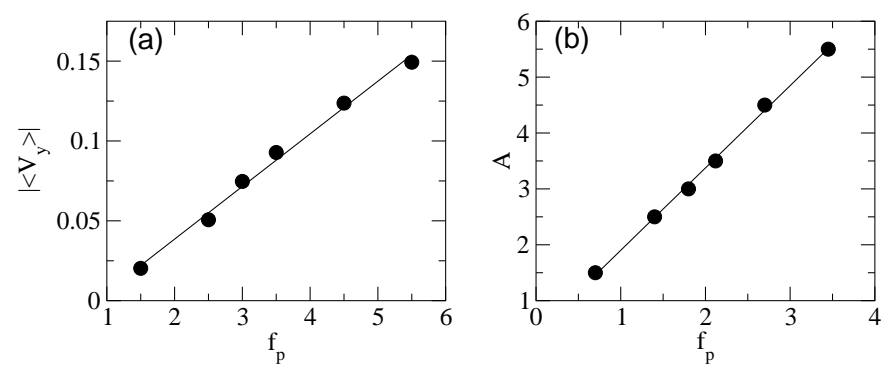

FIG. 9. (a) The maximum value of $\left|\left\langle V_{y}\right\rangle\right|$ vs $f_{p}$ for the system in Fig. 7. The solid line is a linear fit. (b) The value of $A$ at which the maximum in $\left|\left\langle V_{y}\right\rangle\right|$ occurs as a function of $f_{p}$ for the system in Fig. 7. The solid line is a linear fit.
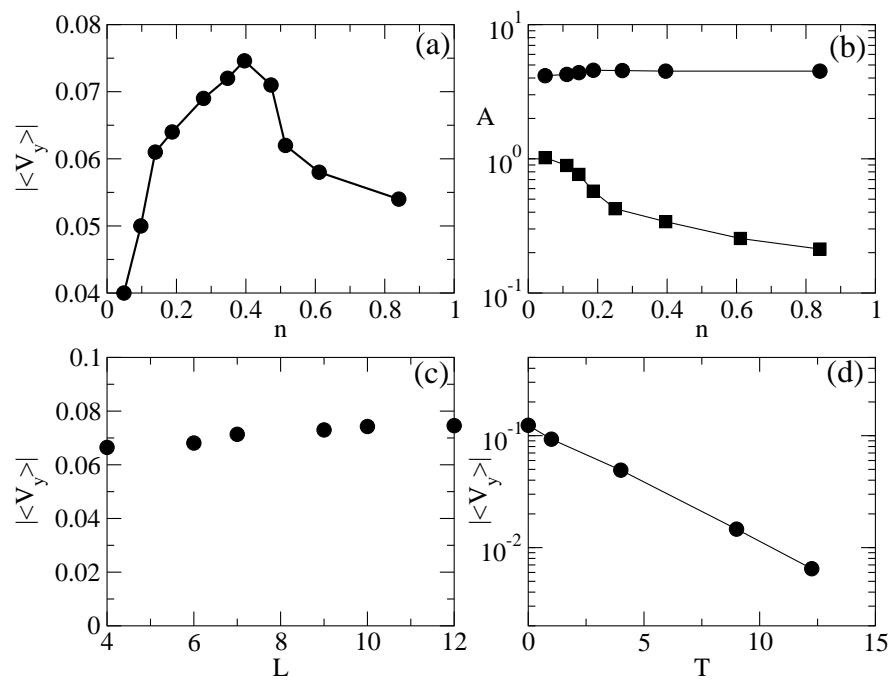

FIG. 10. (a) The maximum value of $\left|\left\langle V_{y}\right\rangle\right|$ vs particle density $n$ for a system with $f_{p}=3.0$. (b) The dynamic phase diagram for $A$ vs $n$. The squares separate the pinned phase from the plastic flow phase and the solid circles separate the plastic flow phase from the reordered moving phase. (c) The maximum in $\left|\left\langle V_{y}\right\rangle\right|$ vs system size $L$ for a system with fixed $n=0.4$ and $f_{p}=3.0$. (d) The maximum value of of $\left|\left\langle V_{y}\right\rangle\right|$ vs $T$ for a system with fixed $n=0.3$ and $f_{p}=3.0$.

lattice start to crystallize and the transverse ratcheting effect becomes very difficult to detect. In Fig. 9(a) we show that the maximum value of $\left|\left\langle V_{y}\right\rangle\right|$ increases linearly with the maximum pinning force and in Fig. 9(b) we show that the value of $A$ where the peak value in $\left|\left\langle V_{y}\right\rangle\right|$ occurs also increases linearly with $f_{p}$.

Next we consider the effects of changing the particle density $n$. We fix both the ratio of particles to pinning sites and the system size. In this way, the effects of the particle-particle interactions can be studied. The transverse ratchet arises due to the interaction between the moving particles and the repulsive force from the pinned particles. If the density of the system is lowered, the average velocity $\left\langle V_{y}\right\rangle$ of the transverse ratchet effect should drop since the particles are further apart. In Fig. 10(a) we plot the peak value for $\left|\left\langle V_{y}\right\rangle\right|$ vs $n$ for a system with $f_{p}=3.0$. For low density, $\left|\left\langle V_{y}\right\rangle\right|$ is small and steadily increasing. $\left|\left\langle V_{y}\right\rangle\right|$ reaches a peak near $n=0.4$ and then gradually decreases for higher $n$. This fall off in $\left|\left\langle V_{y}\right\rangle\right|$ at larger $n$ occurs because the increasing strength of the particle-particle interactions reduces the effectiveness of the pinning, and portions of the lattice crystallize and become rigid at higher values of $n$. In Fig. 9 it was shown that stronger pinning increases the magnitude of the maximum value of $\left|\left\langle V_{y}\right\rangle\right|$ for fixed particle density, highlighting the central role that the pinning plays in the transverse ratchet effect. Another measure of the reduced effective pinning at high densities is shown in Fig. 10(b) where we plot the dynamic phase diagram as a function of $A$ and $n$ for the same system in Fig. 9. The squares mark the separation between the pinned and plastic flow regimes, and the circles separate the plastic flow from the ordered regime. As $n$ increases, the depinning threshold drops and the pinned phase monotonically decreases in extent. The value of $A$ at which the transition to the ordered phase occurs is relatively constant as a function of $n$, but shows a very small peak feature similar to what is seen in Fig. 10(a). This result indicates that the ratchet effect arises due to the collective interactions between the particles, since when the interactions dominate over the pinning, the transverse ratcheting effect is reduced or destroyed.

We next consider finite size effects in a system where we set $n=0.4, f_{p}=3.0$, and fix the ratio of the number of particles to the number of pinning sites. We perform a series of simulations for increasing system size $L$ and plot the maximum value of $\left|\left\langle V_{y}\right\rangle\right|$ vs $L$ in Fig. 10(c). We find only a slight decrease in $\left|\left\langle V_{y}\right\rangle\right|$ at small $L$, and observe that $\left|\left\langle V_{y}\right\rangle\right|$ is constant at higher $L$. This indicates that the transverse ratchet effect is not a finite size effect and that the value of $\left|\left\langle V_{y}\right\rangle\right|$ is determined by the relative density and strength of the pins.

Next we consider the effect of finite temperature. In Fig. 10(d) we plot the maximum value of $\left|\left\langle V_{y}\right\rangle\right|$ vs $T$ for the same system in Fig. 9(a) for $n=0.4$. Here, we find that $\left|\left\langle V_{y}\right\rangle\right|$ fits well to an exponential decay, $\left|\left\langle V_{y}\right\rangle\right| \propto \exp (-\alpha T)$. This indicates an activated transport mechanism. In the plastic flow regime where the transverse ratchet effect occurs, moving particles circulate around pinned particles, but as the temperature increases, the pinned particles become activated out of the pinning sites and the ratchet effect is lost.

We also note that another test of the fact that the pinning density and strength controls the effectiveness of the transverse ratchet is to vary the number of particles while holding the number of pinning sites fixed. As the number of particles increases, the maximum in $\left|\left\langle V_{y}\right\rangle\right|$ stays at roughly the same value (not shown) since the number of pinned particles, which controls the ratchet effect, remains constant. 


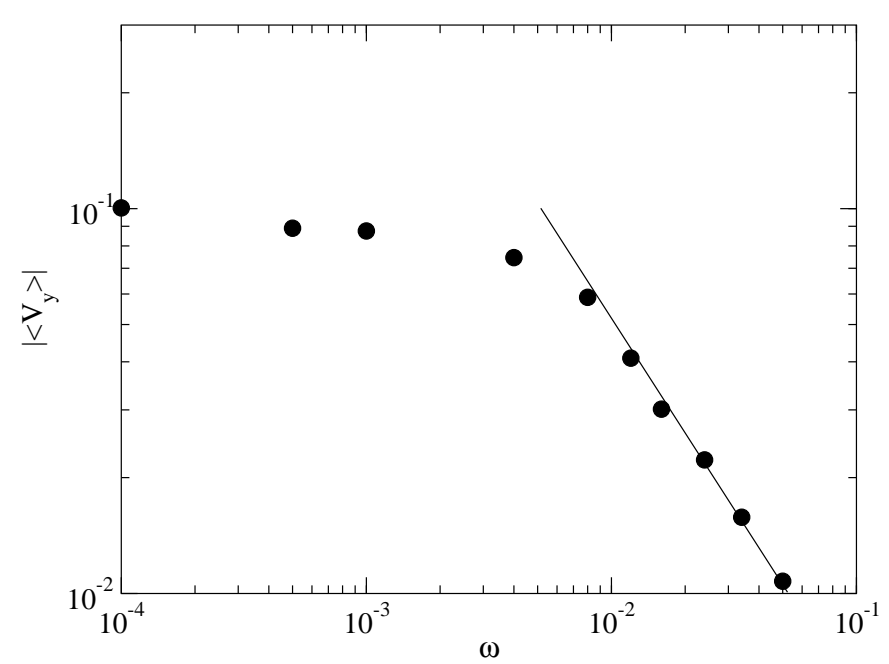

FIG. 11. $\left|\left\langle V_{y}\right\rangle\right|$ vs $\omega$ for fixed $A=2.0, n=0.4$ and $f_{p}=3.0$. The solid line is a fit to $1 / \omega$.

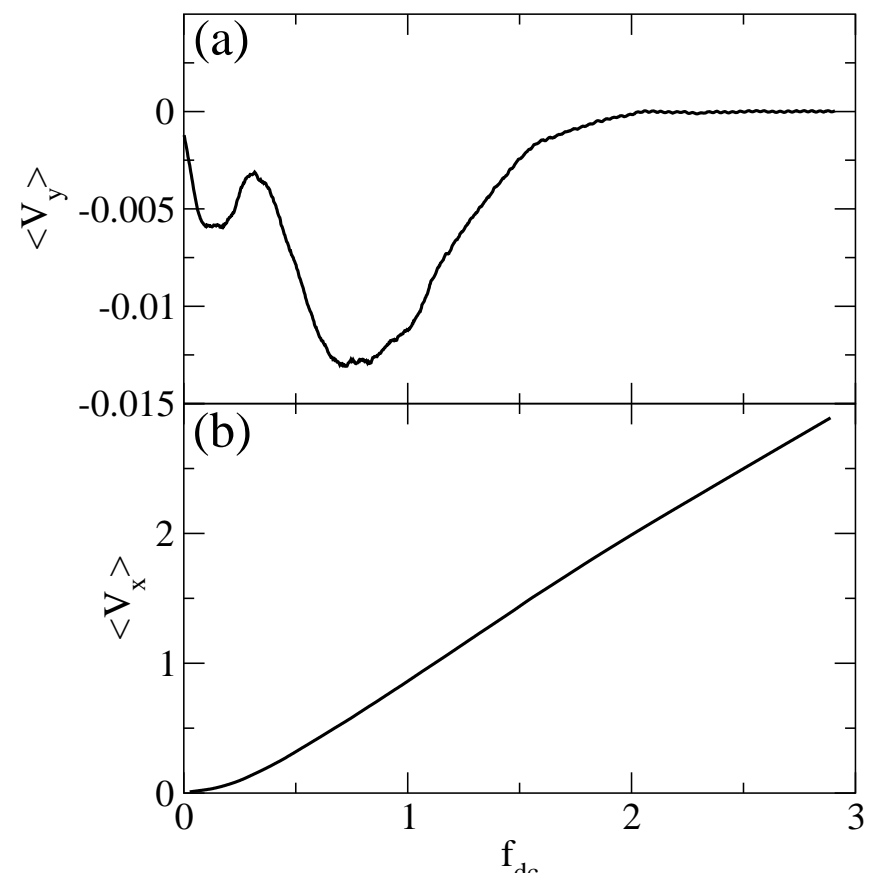

FIG. 12. (a) $\left\langle V_{y}\right\rangle$ vs $f_{d c}$ and (b) $\left\langle V_{x}\right\rangle$ vs $f_{d c}$ for $f_{p}=1.4$, $n=0.4$, and fixed $A=0.5$.

\section{E. Effects of Varied Frequency and dc drive}

In Fig. 11 we show the maximum value of $\left|\left\langle V_{y}\right\rangle\right|$ vs frequency $\omega$ for fixed $A=2.0$ with the same parameters as in Fig. 10 (a) for $n=0.4$. For low frequencies, $\left|\left\langle V_{y}\right\rangle\right|$ saturates to a constant value. For the higher frequencies, $\left|\left\langle V_{y}\right\rangle\right|$ decreases approximately as $1 / \omega$. We note that as the frequency increases, the effective radius of the circular particle orbit decreases. For increasing $A$ and fixed $\omega$,
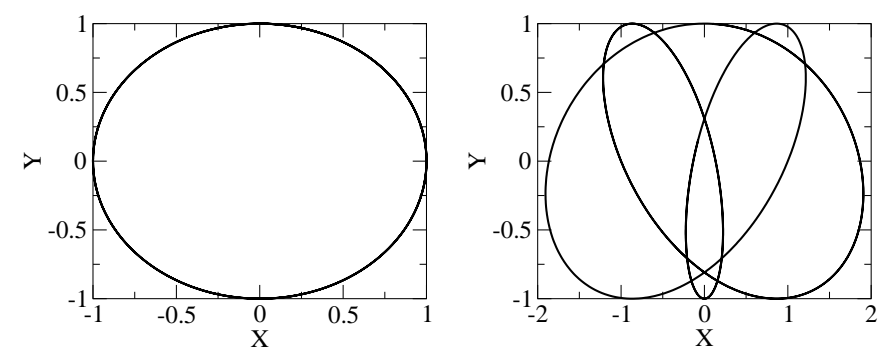

FIG. 13. (a) Orbit for a system with a circular ac drive. (b) Orbit for a system with $g(t)=\cos (6 \omega t) \hat{\mathbf{y}}+[\sin (4 \omega t)+\sin (6 \omega t)] \hat{\mathbf{x}}$, with $\omega=0.001$.

the radius of the orbit increases, and in Fig. 7 we found that $\left|\left\langle V_{y}\right\rangle\right|$ increases linearly with $A$. From these sets of simulations we find that the effective transverse ratchet effect as measured by the maximum value of $\left|\left\langle V_{y}\right\rangle\right|$ goes as $(A / \omega) f_{p} \exp (-\alpha T)$. Also, as a function of $n$, the system shows a maximum in $\left|\left\langle V_{y}\right\rangle\right|$, indicating that there is an optimal value of particle-particle interaction strength for the transverse ratchet effect.

We have also considered the case of fixed $A$ and increasing $f_{d c}$. In general we find a similar transverse ratchet effect and dynamical reordered regime at high $f_{d c}$. In this case, $\left\langle V_{x}\right\rangle$ monotonically increases and there is no overshoot effect. In Fig. 12(a) we plot $\left\langle V_{y}\right\rangle$ vs $f_{d c}$ and in Fig. $12(\mathrm{~b})$ we show $\left\langle V_{x}\right\rangle$ vs $f_{d c}$ for a system with fixed $f_{p}=1.5, A=0.5$, and $n=0.4$. For $f_{d c}>2.0$, the system reorders to a moving crystal. At low drives there is some creep due to the application of the ac drive. As $f_{d c}$ increases, more of the particles become depinned and can ratchet so a peak in $\left\langle V_{y}\right\rangle$ occurs near $f_{d c}=0.9$, while at high drives all particles are depinned and $\left\langle V_{y}\right\rangle$ goes to zero. There is an interesting second peak feature in $\left\langle V_{y}\right\rangle$ at low $f_{d c}$. This occurs near what would be the dc depinning threshold at $A=0.0$. For $f_{p}>3.0$ this second peak structure disappears.

\section{RATCHET EFFECTS WITHOUT DC DRIVES}

If we consider a system with $f_{d c}=0$, it is still possible to obtain a nonzero dc response in either the $x$ or $y$-direction if the closed orbit of the ac drive breaks an additional spatial symmetry. For particles moving on periodic substrates, it was previously shown that a rich variety of phase locking and ratchet effects can occur when the ac drive is noncircular $[16,17]$. Some of these effects include a number of current reversals as the ac amplitude is increased [16]. In the case of a disordered substrate, we find that it is still possible to produce a ratchet effect and even reversals when $f_{d c}=0$ and an acircular ac drive is applied; however, the well defined phase locking steps seen for periodic substrates are missing. In Fig. 13 we show two examples of the closed orbits that a particle would follow in the absence of pinning or other particles. 

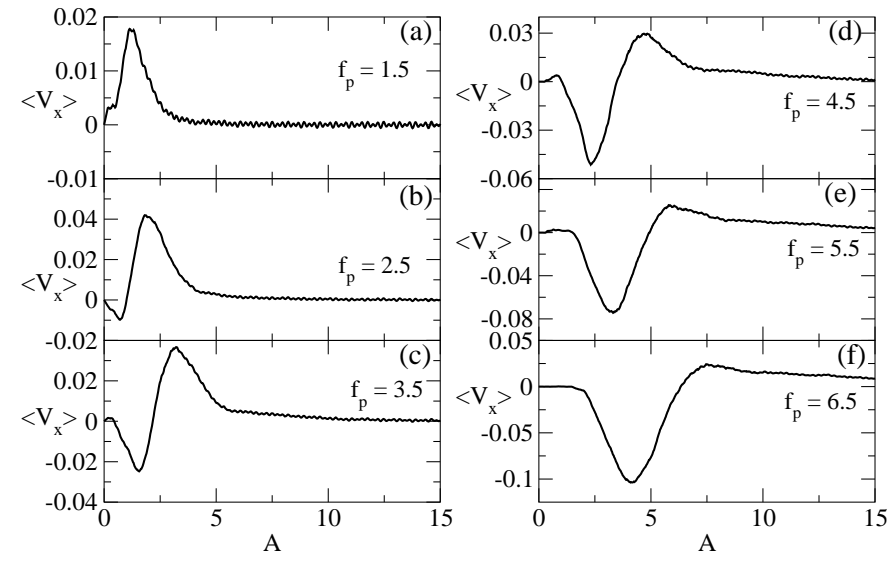

FIG. 14. $\left\langle V_{x}\right\rangle$ vs $A$ for a system driven with the ac drive shown in Fig.13(b) for $f_{p}=$ (a) 1.5, (b) 2.5, (c) 3.5, (d) 4.5, (e) 5.5 , and (f) 6.5 .

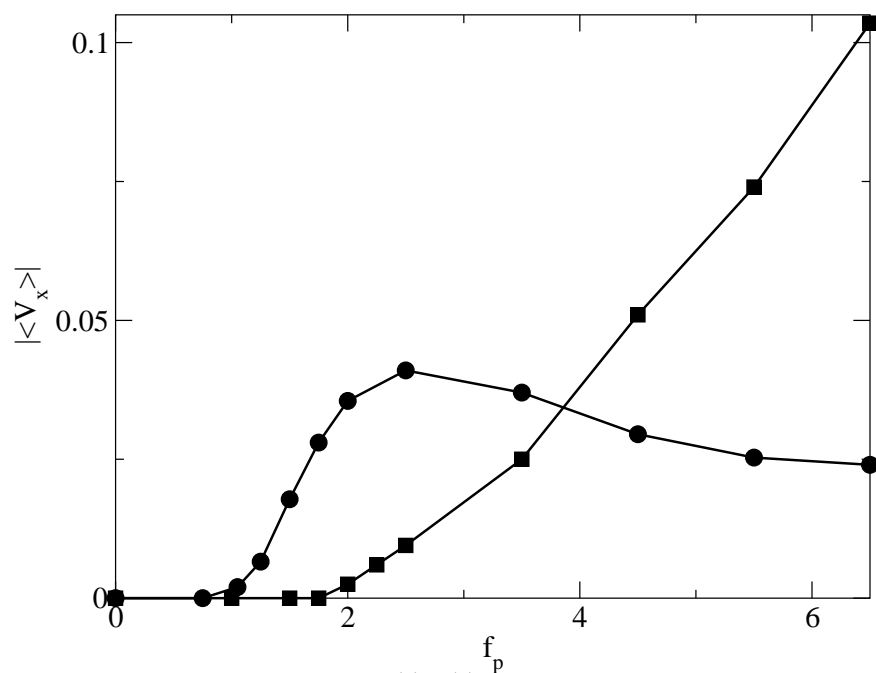

FIG. 15. The maximum $\left|\left\langle V_{x}\right\rangle\right|$ for the initial negative peak (squares) and the positive peak (circles) vs $f_{p}$ for the system shown in Fig. 14.

Figure 13(a) illustrates a circular orbit which does not break spatial symmetry. With only a circular ac drive and no dc drive, the particles do not ratchet. In Fig. 13(b) we show an orbit for $g(t)=A \cos (6 \omega t) \hat{\mathbf{y}}+$ $A[\sin (4 \omega t)+\sin (6 \omega t)] \hat{\mathbf{x}}$, with $\omega=0.001$. Here the reflection symmetry across the $y$ axis is broken. In Fig. 14 we plot $\left\langle V_{x}\right\rangle$ vs $A$ for $f_{d c}=0, n=0.4$, and varied $f_{p}$ for the system with the ac drive shown in Fig. 13(b). In Fig. 14(a) for low $f_{p}=1.5$, there is a positive peak in $\left\langle V_{x}\right\rangle$, indicating that a $+x$ rectification is occurring even the absence of a dc drive. At high $A,\left\langle V_{x}\right\rangle$ goes to zero and the system reorders into a moving crystal. For $f_{p}=2.5$ [Fig. 14(b)], the positive peak in $\left\langle V_{x}\right\rangle$ is larger and there is an additional negative peak in $\left\langle V_{x}\right\rangle$ that occurs before the positive $\left\langle V_{x}\right\rangle$ peak. As $f_{p}$ increases for

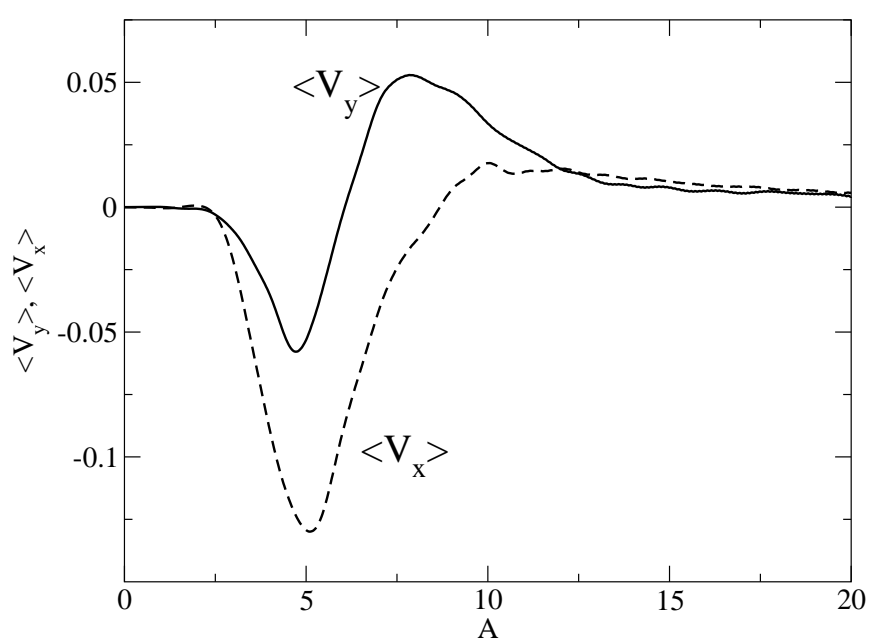

FIG. 16. Ratchet effect in $\left\langle V_{y}\right\rangle$ (solid curve) and $\left\langle V_{x}\right\rangle$ (dashed curve) for the same system in Fig. 14 with $f_{p}=7.0$.

Fig. 14(c-f), the negative peak in $\left\langle V_{x}\right\rangle$ grows in magnitude and the locations of both peaks shift to higher values of $A$. For the higher values of $f_{p}$, there is an initial pinned region where all the particles are trapped and $\left\langle V_{x}\right\rangle=0$. As $f_{p}$ increases, this pinned region grows in width. In Fig. 15 we plot the absolute value of the maximum $\left|\left\langle V_{x}\right\rangle\right|$ for (squares) the initial negative peak and (circles) the subsequent positive peak. Here it can be seen that there is a minimum pinning force of $f_{p} \approx 0.9$ required to produce any kind of ratcheting effect. The positive peak initially grows in magnitude and reaches a maximum value at $f_{p} \approx 2.5$. As $f_{p}$ increases further, the maximum height of the positive peak decreases; however, the range of $A$ over which a positive ratchet effect occurs is also widened as seen in Fig. 14. The negative peak first appears at a higher value of $f_{p}=1.75$ and increases monotonically in size as $f_{p}$ is further increased.

We find that the ratchet effect for asymmetric ac drives can occur in both the $x$ and $y$ directions simultaneously. In Fig. 16 we plot $\left\langle V_{x}\right\rangle$ (dashed curve) and $\left\langle V_{y}\right\rangle$ (solid curve) for the same system as in Fig. 14 with $f_{p}=7.0$. Here, the same trend in $\left\langle V_{x}\right\rangle$ is seen with an initial pinned regime followed by a strong negative peak and a reversal to a positive peak at higher $A$. For $\left\langle V_{y}\right\rangle$, we find a similar trend, with an initial negative peak which begins at the same value of $A$ as the negative peak in $\left\langle V_{x}\right\rangle$. The negative peak for $\left\langle V_{y}\right\rangle$ has a smaller magnitude than the negative peak in $\left\langle V_{x}\right\rangle$. For higher $A$, a much larger positive peak in $\left\langle V_{y}\right\rangle$ occurs near the value of $A$ where $\left\langle V_{x}\right\rangle$ crosses zero. At very high $A$ the ratchet effect in both directions disappears.

These results show that it is possible to obtain a ratchet effect for particles interacting with random disorder where the symmetry breaking comes from the ac drive alone. The current reversals and the magnitude of the ratchet effect persist for varied system size and thus they are not artifacts caused by the system size. 
Additionally, if we repeat the simulations for different random pinning configurations, the same types of curves are produced. We have also tested a number of different ac drives that break a reflection symmetry. The details of the curves are different; however, in general at least one current reversal is observed in each case.

The ratchet effect in the absence of the dc drive is consistent with the observations in earlier studies of particles driven over periodic disorder [16]. In the previous study, crossed ac drives with characteristics similar to those shown in Fig. 13(b) produced dc transport in both the $x$ and $y$ directions. In that system the ratcheting effect occurred in well defined regimes. Additionally, the ratchet effect showed a number of current reversals for increasing ac amplitude; however, for low $A$, most of the steps were in the negative direction, while for higher $A$, most of the steps were in the positive direction. This is consistent with the results obtained here for random disorder where we find a general smearing of all the steps. The ratchet effect in both the periodic and random disorder cases does not occur for ac drives that do not break at least one reflection symmetry.

\section{SUMMARY}

In summary, we have shown that for repulsively interacting particles moving over a disordered substrate, it is possible to obtain a transverse ratchet effect when a circular ac drive is imposed over an additional dc drive. In the absence of the circular ac drive the particles move only in the direction of the dc drive. When the circular ac drive is present, the particles can have an additional dc drift velocity in the direction transverse to the applied dc drive. The average transverse velocity shows a peak as a function of ac amplitude for fixed dc drive or for fixed ac amplitude and increasing dc drive. At high ac or dc drives the system reorders to a moving crystal phase. This phase is distinct from a moving smectic, which would occur if only a dc drive were applied. In the reordered phase the transverse ratchet effect is lost. For fixed dc drive and increasing ac drive we also observe what we term an overshoot effect where the longitudinal velocity is larger than the maximum possible value that could be produced by the dc drive alone. This overshoot effect arises due to a longitudinal ratchet effect produced near the reordering transition where the nonlinearity of the longitudinal velocity force response changes. We analyze the transverse ratchet effect for a wide range of system parameters, including disorder strength, particle density, temperature, and ac frequency. We also show that the ratchet effect is robust for increasing system sizes. The transverse ratchet effect arises due to a symmetry breaking by the chirality of the ac drive plus an additional symmetry breaking by the dc drive. If the crossed ac drives are more complicated, such that the closed orbits themselves are asymmetric, then it is possible to obtain a ratchet effect even in the absence of a dc drive.

Acknowledgments - We thank Z. Toroczkai and M.B. Hastings for useful discussions. This work was supported by the US DOE under Contract No. W-7405-ENG-36.

[1] For a review see: P. Reimann Phys. Rep. 361, 57 (2002); R.D. Astumian and P. Hänggi, Physics Today, 55 (11), 33 (2002).

[2] R.D. Astumian and M. Bier, Phys. Rev. Lett. 72, 1766 (1994); R.D. Astumian, Science 276, 917 (1997); F. Jülicher, A. Ajdari, and J. Prost, Rev. Mod. Phys. 69, 1269 (1997).

[3] C. Marquet, A. Buguin, L. Talini, and P. Silberzan, Phys. Rev. Lett. 88, 168301 (2002); S.H. Lee, K. Ladavac, M. Polin, and D.G. Grier, ibid. 94, 110601 (2005); D. Babic and C. Bechinger, ibid. 94, 148303 (2005).

[4] E. Lundh and M. Wallin, Phys. Rev. Lett. 94, 110603 (2005).

[5] Z. Farkas, P. Tegzes, A. Vukics, and T. Vicsek, Phys. Rev. E 60, 7022 (1999); J.F. Wambaugh, C. Reichhardt, and C.J. Olson, ibid. 65, 031308 (2002); D. van der Meer, P. Reimann, Ko van der Weele, and D. Lohse, Phys. Rev. Lett. 92, 184301 (2004).

[6] H. Linke, T.E. Humphrey, A. Lofgren, A.O. Sushkov, R. Newbury, R.P. Taylor, and P. Omling, Science 286, 2314 (1999); H. Linke, T.E. Humphrey, P.E. Lindelof, A. Lofgren, R. Newbury, P. Omling, A.O. Sushkov, R.P. Taylor, and H. Xu, Appl. Phys. A 75, 237 (2002).

[7] C.S. Lee, B. Jankó, I. Derenyi, and A.L. Barabasi, Nature (London) 400, 337 (1999); J.F. Wambaugh, C. Reichhardt, C.J. Olson, F. Marchesoni, and F. Nori, Phys. Rev. Lett. 83, 5106 (1999).

[8] M.B. Hastings, C.J. Olson Reichhardt, and C. Reichhardt, Phys. Rev. Lett. 90, 247004 (2003); C.J. Olson Reichhardt, C. Reichhardt, M.B. Hastings, and B. Jankó, Physica C 404, 266 (2004).

[9] C.J. Olson, C. Reichhardt, B. Jankó, and F. Nori, Phys. Rev. Lett. 87, 177002 (2001); V.K. Vlasko-Vlaskov et al., to be published.

[10] B.Y. Zhu, F. Marchesoni, V.V. Moshchalkov, and F. Nori, Phys. Rev. B 68, 014514 (2003).

[11] R. Wördenweber, P. Dymashevski, and V.R. Misko, Phys. Rev. B 69, 184504 (2004).

[12] J.E. Villegas, E.M. Gonzalez, M.P. Gonzalez, J.V. Anguita, and J.L. Vicent, Phys. Rev. B 71, 024519 (2005); J. Van de Vondel, C.C. de Souza Silva, B.Y. Zhu, M. Morelle, and V.V. Moshchalkov, Phys. Rev. Lett. 94, 057003 (2005).

[13] F. Falo, P.J. Martinez, J.J. Mazo, and S. Cilla, Europhys. Lett. 45, 700 (1999); E. Trias, J.J. Mazo, F. Falo, and T.P. Orlando, Phys. Rev. E 61, 2257 (2000); G. Carapella and G. Costabile, Phys. Rev. Lett. 87, 077002 (2001); D.E. Shalóm and H. Pastoriza, ibid. 94, 
177001 (2005).

[14] J.B. Majer, J. Peguiron, M. Grifoni, M. Tusveld, and J.E. Mooij, Phys. Rev. Lett. 90, 056802 (2003); A.V. Ustinov, C. Coqui, A. Kemp, Y. Zolotaryuk, and M. Salerno, ibid. 93, 087001 (2004).

[15] C. Reichhardt, C.J. Olson, and M.B. Hastings, Phys. Rev. Lett. 89, 024101 (2002); Phys. Rev. E. 69, 056115 (2004).

[16] C. Reichhardt and C.J. Olson Reichhardt, Phys. Rev. E 68, 046102 (2003).

[17] R. Guantes and S. Miret-Artés, Phys. Rev. E. 67, 046212 (2003); S. Sengupta, R. Guantes, S. Miret-Artes, and P. Hänggi, Physica A 338, 406 (2004).

[18] M.A. Makeev, I. Derényi, and A.L. Barabási, Phys. Rev. E 71, 026112 (2005).

[19] N. Grønbech-Jensen, Int. J. Mod. Phys. C 7, 873 (1996).

[20] S. Bhattacharya and M.J. Higgins, Phys. Rev. Lett. 70, 2617 (1993).

[21] A.E. Koshelev and V.M. Vinokur, Phys. Rev. Lett. 73, 3580 (1994).

[22] K. Moon, R.T. Scalettar, and G.T. Zimányi, Phys. Rev. Lett. 77, 2778 (1996); C.J. Olson, C. Reichhardt, and F. Nori, ibid. 81, 3757 (1998); A.B. Kolton, D. Domínguez, and N. Grønbech-Jensen, ibid. 83, 3061 (1999).

[23] P. Le Doussal and T. Giamarchi, Phys. Rev. B 57, 11356 (1998); L. Balents, M.C. Marchetti, and L. Radzihovsky, ibid. 57, 7705 (1998).

[24] C. Reichhardt and C.J. Olson, Phys. Rev. Lett. 89, 078301 (2002).

[25] D.S. Fisher, Phys. Rep. 301, 113 (1998).

[26] K. Saunders, J.M. Schwarz, M.C. Marchetti, and A.A. Middleton, Phys. Rev. B 70, 024205 (2004).

[27] S.O. Valenzuela, Phys. Rev. Lett. 88, 247003 (2002). 